Silva Arciniega, María del Rosario (2016) Dimensiones Psicosociales de la Pobreza: Percepción de una realidad recuperada. Universidad Nacional Autónoma de México. 2a. Edición. México.

ISBN: 978-607-02-8581-3

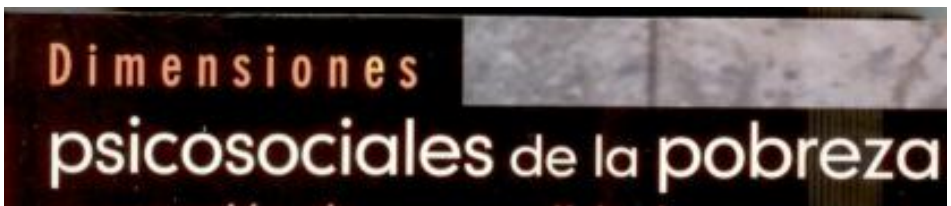
percepción de una realidad recuperada

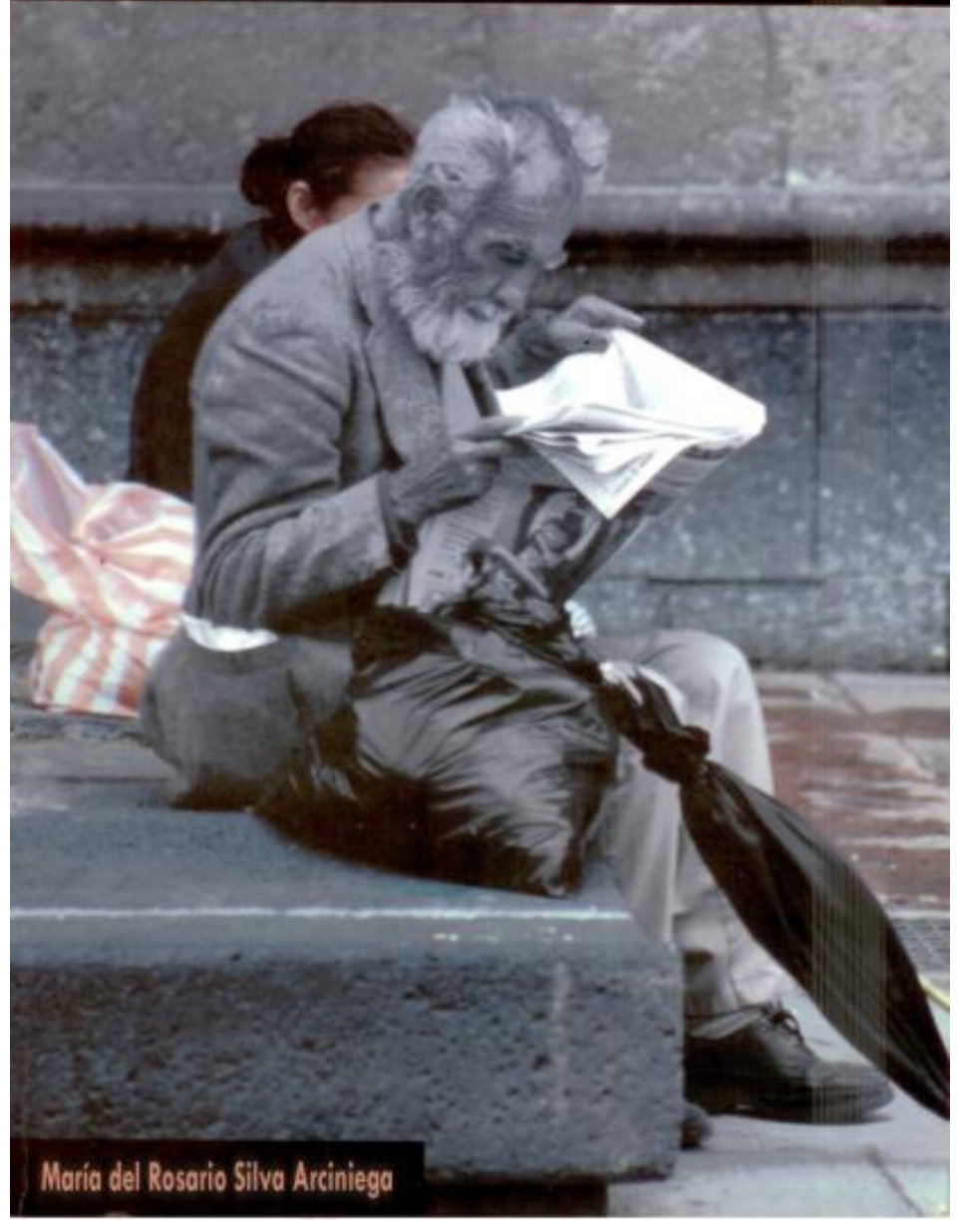

RESEÑA POR

Dra. María del Rosario Silva Arciniega 


\section{DIMENSIONES PSICOSOCIALES DE LA POBREZA: PERCEPCIÓN DE UNA REALIDAD RECUPERADA}

María del Rosario Silva Arciniega ${ }^{1}$

\section{PROEMIO}

Este texto surgió ante la necesidad que como trabajadora social sentía de realizar una investigación en donde se pudiera recuperar la voz de los pobres, intentando identificar su manera de sentir, pensar y discernir acerca de lo que significa la pobreza para ellos; ya que durante la interacción que con personas en estado de carencia y/o vulnerabilidad he vivido, sobre todo cuando impartía en la ENTS, UNAM alguna de las Prácticas Comunitaria o Institucional, que me permitieron vivir experiencias en donde aparentemente quien pudiera definirse como "pobre" desde alguno de los paradigmas establecidos - línea de pobreza, necesidades básicas insatisfechas, o cualquiera otro - terminaban aportando sus propios conocimientos, destrezas e historias de vida, desde donde se percibía una riqueza extraordinaria, así como una fuerte capacidad de resiliencia.

Desde ahí nació mi deseo por conocer una manera diferente de medir y definir a la pobreza, una forma que no fuera solo la de los haberes, sino que incluyera los sentires y pensares. La tarea no fue sencilla, implicó varios años de investigación que fructificaron en: una primera aproximación que permitió detectar 12 DPP para la CDMX emanadas de la aplicación de análisis factorial de tipo Alpha con rotación varimax; en una segunda aproximación que implicó la administración del instrumento en muestras propositivas de siete Estados de la República, se pudo identificar cómo desde la aplicación del constructo se detectaba la problemática emergente de cada localidad; la tercera y cuarta aproximaciones implicaron la detección de 10 DPP y

\footnotetext{
${ }^{1}$ Dra. Ma. del Rosario Silva Arciniega, Escuela Nacional de Trabajo Social, Universidad Nacional Autónoma de México, rsilva@unam.mx
} 
la construcción de un Modelo Factorial Confirmatorio de la Escala de Pobreza para la CDMX y Provincia, la quinta llevó a la estandarización de la Escala de Pobreza.

CONCEPTOS CLAVE: Pobreza, Dimensiones psicosociales, Percepción

\section{RESEÑA}

La razón que llevó a realizar el estudio sobre las Dimensiones Psicosociales de la Pobreza ${ }^{2}$, en la Escuela Nacional de Trabajo Social de la Universidad Nacional Autónoma de México, radicó en el hecho de que el hacer de los trabajadores sociales fundamentalmente se encuentra dirigido hacia las personas en estado de carencia y vulnerabilidad.

En el año en que se inició la investigación (1994), los teóricos de la pobreza la medían en casi todos los casos desde variables eminentemente cuantitativas, no era usual recuperar la voz de los que la padecían, ni rescatar o identificar de manera simultánea la carencia y la riqueza que sin duda poseen todas las personas; de tal manera que este estudio fundamenta su relevancia en el hecho de identificar de la manera de sentir, pensar y discernir acerca de lo que significa la pobreza desde quienes la padecen.

El texto es producto de la realización de una investigación polietápica es decir, realización de varios estudios:

1) Estudio exploratorio de campo transversal cuyo objetivo fue obtener un instrumento válido y confiable capaz de medir las Dimensiones Psicosociales de la Pobreza (DPP). Se aplicó una red semántica de los conceptos "Pobreza" y "Riqueza", a una muestra no propositiva de 52 personas de la Colonia Tlalmille, en el espacio donde se realizaba la Práctica Comunitaria bajo mi

\footnotetext{
${ }^{2}$ Estudio financiado por el PAPIIT y el CONACYT.
} 
supervisión, a los estudiantes del $5^{\circ}$ semestre de la Licenciatura en Trabajo Social. Se obtuvieron 159 palabras generadoras de pobreza, las que tuvieron peso más fuerte fueron: Carencia 82 puntos, Escasez 48, Limitaciones 48, Dinero 45 y Necesidades 44; las palabras se integraron en 17 categorías a partir de la sinonimia y fueron: Carencia y pobreza, Tristeza, Trabajo, Dinero, Vivienda y servicios, Enfermedad, Instrucción y Cultura, Humildad, Felicidad, Alimentación, Dificultad, Convivencia, Atuendo, Delincuencia, Anhelos, Vergüenza y Hogar. Con base en las palabras encontradas, se localizaron las frases obtenidas en las respuestas a los reactivos abiertos recuperados de los instrumentos de medición y se transformaron en los aciertos que integraron la escala de medición de tipo Likert elaborada exprofeso para buscar las DPP. Producto.- Una vez administrada la escala de medición de tipo Likert en la CDMX a 588 personas, se realizaron los procesos estadísticos a partir de la aplicación de análisis factorial de tipo Alpha con rotación varimax, así como del Alpha de Cronbach respectivamente, para obtener validez y confiabilidad de constructo.

2) Estudio descriptivo, de campo y transversal administrado a una muestra propositiva de 2,702 de adultos en las dieciséis Delegaciones de la CDMX. Producto.- Conceptualización de las DPP que a la letra dice: Se entiende por Dimensiones Psicosociales de la Pobreza a los doce factores propios con valor eigen superior a 1.0 obtenidos de la aplicación de análisis factorial de tipo Alpha con rotación varimax y cuyo Alpha de Cronbach fue de 0.9554 . Los factores fueron denominados: Carencia vs Mesura, Control interno vs fatalismo, Aflicción, Asertividad pública, Imagen del yo, Angustia y coraje, Esperanza, Atribución a la pobreza, Dignidad, Acceso a la Cultura, Familia y Aspiración de logro. Este primer intento por localizar las DPP fue fructífero e indicó que se estaba en el camino correcto.

3) Estudio descriptivo de campo transversal aplicado a una muestra propositiva de 4,634 personas provenientes de Oaxaca, Quintana Roo, Guerrero, Hidalgo, Michoacán, Durango y 
Sonora. El resultado obtenido para cada uno de ellos, permitió localizar como primer factor el problema emergente más acuciante de la localidad, es decir, señala las variables específicas sobre las que las autoridades competentes pueden trabajar para aminorar el estado de pobreza de la población, a partir de la generación de planes, programas y proyectos de intervención multidisciplinarios; tal es el caso, por ejemplo, de Oaxaca donde el primer factor fue Hambre, ${ }^{3}$ tal y como ha sido abordada por instituciones como la Secretaría de Desarrollo Social (SEDESOL).

4) Análisis Factorial Confirmatorio para la CDMX y Provincia en donde se determina la existencia de 10 DPP para México denominadas: Seguridad Básica vs Inseguridad, Dinero, Acceso a la cultura, Conformismo, Esperanza, Familia, Hambre, Atribuciones y Creencias, Trabajo y Rechazo a la pobreza, que en su conjunto explican el $52 \%$ de varianza y tienen un Alpha estandarizada para los 43 asertos que componen la escala de 0.97 . El estudio cumplió sus objetivos plenamente.

5) Generación de Modelo Factorial Confirmatorio de la Escala de Pobreza ${ }^{4}$ se aislaron dos factores ${ }^{5}:$ 1) Factores internos de la pobreza que integran las dimensiones denominadas: Atribuciones y creencias, Conformismo, Seguridad Básica, y Familia; 2) Factores externos de la pobreza que integran las dimensiones denominadas Dinero y Trabajo. Los factores internos son protectores contra la pobreza, se trata de un cúmulo de circunstancias personales y de retroalimentación con los otros, que unidas intervienen para favorecer o perjudicar la interacción del pobre consigo mismo y con su entorno. Los factores externos de la pobreza son los determinantes que afectan al individuo y lo ponen en situación de bonanza o carencia económi-

\footnotetext{
${ }^{3}$ El resultado del estudio en el interior de la República no se publicó en este texto, ya que por sí mismo era muy extenso, se integró a la información obtenida en la CDMX realizar las fases sucesivas del estudio.

${ }^{4}$ Elaborado por el Dr. Jorge Ameth Villatoro.

${ }^{5}$ Originalmente, se hipotetizó que de existir DPP, las Dimensiones Psico generarían factores de internalidad, es decir donde el comportamiento depende de los individuos, y las Dimensiones Sociales generarían factores de externalidad, es decir en donde el control del comportamiento no depende de los individuos.
} 
ca. Estas dos grandes esferas dan cuenta de la forma de sentir, vivir y percibir la pobreza, desde quienes la padecen.

6) Estandarización de la Escala de Pobreza para la publicación de esta segunda edición del texto, se realizó la estandarización de la Escala de Pobreza ${ }^{6}$. Se entiende por estandarización el proceso mediante el cual se convierten las calificaciones de una prueba en una distribución normal, para ello, se define el promedio como el punto central de la distribución y a partir de este punto se agrupan los valores por medio de desviaciones estándar, considerando tres desviaciones hacia arriba y tres hacia abajo. Para la estandarización se utilizó la base de datos de 7,340 sujetos creada durante esa investigación, cuya finalidad radicó en crear un modelo normalizado de la pobreza. La normalización del modelo tiene como objetivo el determinar el grado de pobreza existente en quienes contesten el cuestionario de 43 ítems.

Finalmente se encontró que el pobre de acuerdo con los resultados de este estudio es un ser digno, que afronta con coraje y arrojo los avatares cotidianos de sus carencias económicas, fundamentalmente ocasionadas por falta de trabajo y porque éste, de tenerlo no es adecuadamente remunerado. El pobre que encontramos, es una persona íntegra gracias a la riqueza de sus atribuciones y creencias, su seguridad básica, su no conformismo y fundamentalmente a la vivencia de las positivas experiencias afectivas que vivió desde el seno familiar que le nutren y le impulsan a seguir luchando a pesar de sus carencias y privaciones.

\footnotetext{
${ }^{6}$ La estandarización fue apoyada por el Lic. Rafael Zepeda Barrios.
} 